\title{
Predictors of folate status among pregnant Japanese women: the Hokkaido Study on Environment and Children's Health, 2002-2012
}

\author{
Thamar A. Yila ${ }^{1}$, Atsuko Araki ${ }^{1}$, Seiko Sasaki ${ }^{2}$, Chihiro Miyashita ${ }^{1}$, Kumiko Itoh $^{2}$, Tamiko Ikeno ${ }^{1}$, \\ Eiji Yoshioka ${ }^{3}$, Sumitaka Kobayashi ${ }^{2}$, Houman Goudarzi ${ }^{1}$, Toshiaki Baba ${ }^{2}$, Titilola Braimoh ${ }^{1}$, \\ Hisanori Minakami ${ }^{4}$, Toshiaki Endo ${ }^{5}$, Kazuo Sengoku ${ }^{6}$ and Reiko Kishi ${ }^{1 *}$ \\ ${ }^{1}$ Hokkaido University Center for Environmental and Health Sciences, Sapporo 060-0812, Japan \\ ${ }^{2}$ Department of Public Health Sciences, Hokkaido University Graduate School of Medicine, Sapporo 060-8638, Japan \\ ${ }^{3}$ Department of Health Sciences, Asahikawa Medical University, Asabikawa 078-8510, Japan \\ ${ }^{4}$ Department of Obstetrics and Gynecology, Hokkaido University Graduate School of Medicine, Sapporo 060-8638, Japan \\ ${ }^{5}$ Department of Obstetrics and Gynecology, School of Medicine, Sapporo Medical University, Sapporo 060-8556, Japan \\ ${ }^{6}$ Department of Obstetrics and Gynecology, Asabikawa Medical University, Asabikawa 078-8510, Japan
}

(Submitted 25 April 2015 - Final revision received 2 March 2016 - Accepted 21 March 2016)

\section{Abstract}

The International Clearinghouse for Birth Defects, Surveillance and Research reports a rise in the prevalence rate of spina bifida in Japan. We determined first-trimester folate status of Hokkaido women and identified potential predictors. Participants were 15266 pregnant women of the Hokkaido Study on Environment and Children's Health Cohort. Data were extracted from self-reported questionnaires and biochemical assay results. Demographic determinants of low folate status were younger maternal age (adjusted OR (AOR) 1.48; 95\% CI 1.32, 1.66), lower educational level (AOR 1.27; $95 \%$ CI 1.17, 1.39) and lower annual income (AOR 1.11; $95 \%$ CI 1.01, 1.22). Plasma cotinine concentrations of $1 \cdot 19-65.21 \mathrm{nmol} / \mathrm{l}$ increased the risk of low folate status (AOR 1.20; $95 \%$ CI 1.10, 1.31) and concentrations $>65 \cdot 21 \mathrm{nmol} / \mathrm{l}$ further increased the risk (AOR 1.91; $95 \%$ CI 1·70, 2.14). The most favourable predictor was use of folic acid (FA) supplements (AOR 0.19; $95 \%$ CI $0 \cdot 17,0 \cdot 22$ ). Certain socio-demographic factors influence folate status among pregnant Japanese women. Modifiable negative and positive predictors were active and passive tobacco smoking and use of FA supplements. Avoiding both active and passive tobacco smoking and using FA supplements could improve the folate status of Japanese women.

\section{Key words: Folate status: Pregnancy: Tobacco smoking: Hokkaido Study on Environment and Children's Health}

Folate as a cofactor in one-C metabolism is essential for all cellular processes, especially in conditions of rapid cell replications and tissue growth, such as pregnancy. The role of synthetic folic acid (FA) supplements in the prevention of neural tube defects (NTD) has been well documented ${ }^{(1,2)}$. Several countries with policies of FA food fortification have reported a $30 \cdot 0-70 \cdot 0 \%$ reduction in the incidence of $\mathrm{NTD}^{(3)}$. As reported by the International Clearinghouse for Birth Defects, Surveillance and Research, Japan has experienced an increase in the prevalence of spina bifida over the past few decades. Although in countries such as the USA and England the prevalence of NTD is about $3 / 10000$ births, Japan has a prevalence of $5 \cdot 2^{(4,5)}$. Efforts have been made to determine folate status of pregnant Japanese women in other regions of Japan but not in Hokkaido ${ }^{(6-9)}$. However, probable small sample sizes, different folate assay techniques and varied folate status definitions/cut-off levels might have yielded inconsistent results. For instance, three previous studies have reported a wide range of folate status among pregnant Japanese women. A study from Aichi Prefecture that defined normal $v$. inadequate plasma folate status as $\geq 6.80$ and $<6.80 \mathrm{nmol} / 1$, respectively, reported folate inadequacy in $1.0 \%$ of forty-one pregnant and 154 non-pregnant participants ${ }^{(6)}$. Another study from Tokyo metropolis among pregnant women in all trimesters reported low serum folate status in $67.0 \%$ of 118 women in their first trimester and $79.3 \%$ overall. The study defined normal, low and deficient folate statuses as having $>13 \cdot 60,6 \cdot 80-13.60$ and $<6.80 \mathrm{nmol} / 1$, respectively ${ }^{(8)}$. The third study from Tokyo metropolis was conducted among fifty-eight young university women. Normal serum folate status was categorised as having $\geq 13.60 \mathrm{nmol} / 1$, low status as having $6.80-13.59 \mathrm{nmol} / 1$ and folate deficiency as having $<6 \cdot 80 \mathrm{nmol} / 1$ of folate concentration. Results showed $12.1 \%$ had folate deficiency and $58.6 \%$ had low folate status ${ }^{(9)}$. In Hokkaido Prefecture, such reports are scarce.

Abbreviations: AOR, adjusted OR; ETS, environmental tobacco smoke; FA, folic acid; NTD, neural tube defects.

* Corresponding author: R. Kishi, fax +81 11706 4725, email rkishi@med.hokudai.ac.jp 
We recently conducted a genetic study using the first part of this cohort's data. Low folate status was reported among $28.7 \%$ of the study population ( $n$ 1784), but the scope of the study excluded a detailed exploration of the demographic and lifestyle predictors of folate status ${ }^{(10)}$. In contrast to previous smaller studies in Japan, this study uses data from a large cohort to explore the various demographic and lifestyle factors that may influence first-trimester folate status of Japanese women in Hokkaido.

\section{Methods}

\section{Study location and population}

Participants of this study were pregnant women recruited during their first trimester ( $<13$ weeks of gestation) from thirtyseven healthcare facilities across Hokkaido Prefecture. They are participants of the ongoing, large-scale birth cohort of the Hokkaido Study on Environment and Children's Health. The study is broadly aimed at observing the health effects of intrauterine exposures to various environmental and genetic factors on fetal development, outcomes of pregnancy and subsequent childhood health. Details of the study have been described elsewhere $^{(11,12)}$. In brief, the ongoing, large-scale cohort started in 2002, with a full-blown, large-scale version from February 2003. A total of 20816 women were recruited between 2002 and March 2012. All pregnant Japanese women who presented at any of the participating healthcare facilities for prenatal care during their first trimester were considered eligible for the study. However, only those who agreed to participate in the study were contacted and recruited. Data were generated from these participants by means of baseline questionnaires,

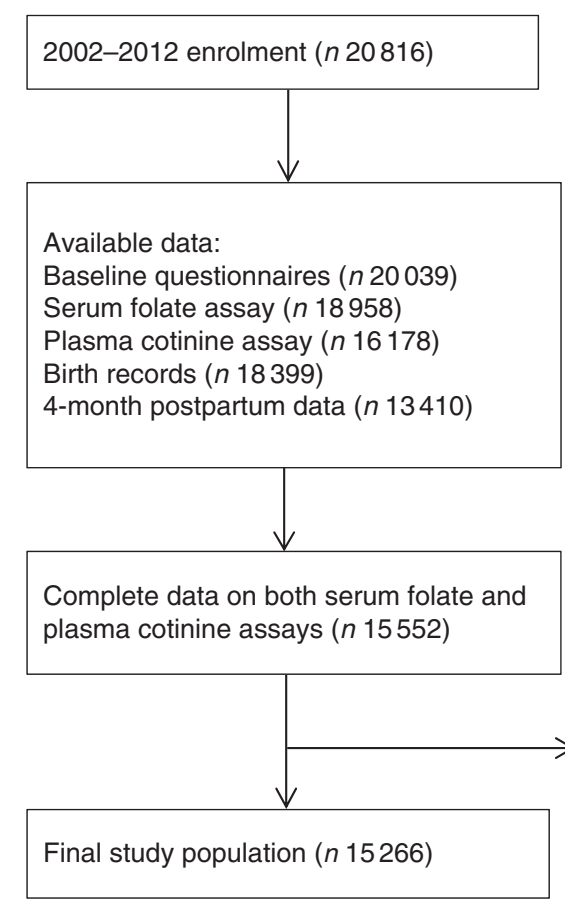

biochemical assays, hospital birth records and 4-month postpartum health records. We finally used a total of 15266 participants; details of the selection process are shown in the flowchart (Fig. 1).

Certain repeated self-reported information obtained from birth records and postpartum questionnaires were compared with baseline questionnaires in order to improve quality and reduce missing data in the whole data set. Otherwise, these data were not used in the analysis of this report. The Institutional Ethical Board for Human Gene and Genome studies at Hokkaido University Graduate School of Medicine approved the study protocol.

\section{Biochemical assays}

Non-fasting, whole blood samples were collected from participants, pre-treated and serum samples were obtained. Serum samples were stored promptly at $4^{\circ} \mathrm{C}$ until they were transported on ice to a commercial laboratory (SRL Corporation Inc.) for folate assay. The ADVIA Centaur Folate Assay Protocol is one of the automated competitive protein binding Immunoassay Technologies. Folate is quantified by direct chemiluminescent acridinium ester technology ${ }^{(13)}$. This technique has an acceptable imprecision of $<10.0 \%$, with an advanced quality control package. It has an analytical sensitivity of $0.91 \mathrm{nmol} / \mathrm{l}$. It can detect from small volumes of as low as $10 \mathrm{ul}$ of biological specimen, making it a method of choice in large epidemiological studies ${ }^{(14)}$. Specimen preparations, shipping and assays were carried out in batches, depending on new recruitments. All laboratory analysts were blinded to participant information. As there is no standard classification of folate status from automated immunoassay techniques, we
Excluded ( $n$ 286):

Diabetes mellitus ( $n$ 90)

Essential hypertension ( $n$ 68)

Inflammatory bowel disease $(n 25)$

Psoriasis $(n 7)$

Hypothyroidism ( $n$ 14)

Epilepsy ( $n$ 32)

Drugs interfering with folate metabolism ( $n 50)$

Fig. 1. Study selection chart: the Hokkaido Study on Environment and Children's Health, 2002-2012, Japan. 
adopted the WHO classification guidelines ${ }^{(15)}$. Nicotine is the toxic chemical in tobacco products and its predominant metabolite is cotinine. Cotinine can be detected in biological specimens as a biomarker of exposure to tobacco. In this study, we used plasma cotinine concentrations to quantitatively classify active and passive smoking status. The details of measurements of plasma cotinine are described in our previous report ${ }^{(16)}$.

\section{Definition of variables}

The dependent variable was folate status. Folate status was classified as follows: folate deficiency $(<6.80 \mathrm{nmol} / \mathrm{l})$, suboptimal status $(6 \cdot 80-13.59 \mathrm{nmol} / \mathrm{l})$ and optimal folate status $(\geq 13.60 \mathrm{nmol} / \mathrm{l})^{(15)}$. Folate deficiency was reported in $0.5 \%$ of the study population. To improve study power, and because non-fasting serum was used for folate assay, we merged this group with the suboptimal category. Active and passive exposure to tobacco smoking statuses were classified based on plasma cotinine cut-off points established in a previous report $^{(16)}$. A non-smoker was defined as having plasma cotinine concentrations of $<1.19 \mathrm{nmol} / 1$, a person exposed to environmental tobacco smoke (ETS) as having $1.19-65.21 \mathrm{nmol} / \mathrm{l}$ and an active smoker as having $>65.21 \mathrm{nmol} / 1$ of plasma cotinine concentration. Prenatal FA supplement use was defined as 'a report on the use of FA supplements before or after conception'. Other nutritional supplements use was defined as 'any report on intake of nutritional supplements other than FA, before or after conception'. Ingestion of alcoholic beverages was categorised based on frequency of intake: monthly, weekly or daily. Self-reported active tobacco smoking was categorised based on the number of cigarette sticks smoked per day - light smokers ( $<10$ cigarette sticks/d), moderate smokers (10-19 cigarette sticks/d) and heavy smokers ( $\geq 20$ cigarette sticks/d). ETS exposure at home was defined as 'living with one or more active smokers'. ETS at workplace referred to 'working with one or more active smokers at workplace'. In this study, the lifestyle habits considered were alcoholic beverage consumption, nutritional supplements use and tobacco use. Potential predictors of folate status were identified based on previous reports. In this study, year of enrolment, maternal age, parity, BMI, educational level, household income, occupation, use of nutritional supplements, active and passive cigarette smoking, alcohol intake, season of the year and geographical location were identified as putative predictors.

\section{Statistical analyses}

Statistical tests of associations included Pearson's $\chi^{2}$ tests and Fisher's exact tests for categorical variables. Skewed serum folate and plasma cotinine concentrations were log-transformed during the preliminary descriptive analyses, thereafter backtransformed. Differences in mean folate levels were explored using ANOVA with post hoc analyses to correct for multiple comparisons. However, the main regression analyses were performed using qualitative folate status. We imputed the missing values present in the data via multivariate imputation by chained equations (MICE), as implemented in the R package mice, obtaining $m=10$ imputed data sets. MICE is a Markov chain Monte Carlo method that uses the correlation structure of the data and imputes missing data values for each incomplete variable $m$ times by regression of incomplete variables on the other available variables iteratively. We used Bayesian logistic regression and fitted the model to the $m=10$ imputed data set, with dichotomised folate status as the outcome variable, and the following as potential predictor variables: age, BMI, parity, educational level, income, occupation, region, year of enrolment, season of the year at enrolment, FA supplements use, other nutritional supplements use, alcohol intake, active cigarette smoking and exposure to ETS both at home and at workplace. We used results of plasma cotinine concentration to quantitatively classify active smoking and passive exposure to tobacco products and regressed against folate status, with adjustment for all other potential predictors. We reported pooled estimates for the main effects of the predictor variables in the model. $P$ values for testing the presence of a linear trend are also reported for predictor variables with more than two categories. Reported effects, CI and $p$ values are pooled over the $m=10$ imputed data sets. In addition, we reported the value of the McFadden's pseudo $R^{2}$ pooled over these data sets. All statistical analyses were performed using JMP 11 Pro Statistical Software Package (SAS), except for the binary logistic regression model, which required multiple imputation of missing data and was performed using $\mathrm{R}$ version 3.2.2. An $\alpha$ level of significance was set at $<0 \cdot 05$.

\section{Results}

Overall, the geometric mean of serum folate concentration was 17.77 (sD 3.58) nmol/l. Among women with optimal folate status, the geometric mean was 20.67 (sD 3.26) nmol/l and was $10 \cdot 83(2.65) \mathrm{nmol} / \mathrm{l}$ among participants with suboptimal folate status. One-sided lower-limit tolerance interval at $95 \%$ of the population was $8.47 \mathrm{nmol} / \mathrm{l}$. Prevalence of folate deficiency was $0.5 \%$. Suboptimal folate status constituted $25.7 \%$, whereas optimal folate status was reported in $73.8 \%$ of the population (Table 1). Initial descriptive analyses using folate as a continuous variable revealed that mean serum folate concentrations increased with increasing maternal age $(P<0 \cdot 001)$, educational status $(P<0.001)$, annual income $(P<0 \cdot 001)$, FA supplements use $(P<0.001)$ and other nutritional supplements use $(P<0.001)$. Mean serum folate concentrations decreased with increasing number of cigarette sticks smoked per day $(P<0 \cdot 001)$, ETS exposure at home $(P<0 \cdot 001)$ and increasing plasma cotinine concentrations $(P<0 \cdot 001)$. Exposure to ETS at both home and work was associated with low folate status, $P<0.001$. About $60.0 \%$ of those with folate deficiency were exposed to ETS both at home and at workplace. Other associations were geographical region, year of enrolment into the study and season of the year (data not shown). Serum folate inversely correlated with plasma cotinine concentration ( $r-0 \cdot 2000, P<0 \cdot 001$, data not shown). Significant differences were observed in mean plasma cotinine concentrations between non-users of FA supplements and users, with geometric means of 46.41 (SD 23.23) nmol/1 and $25 \cdot 27$ (sD 15.32 ) nmol/l, $P<0 \cdot 001$, respectively. In addition, geometric means for non-users and users of other nutritional supplements were 42.49 (sD 21.91) nmol/l and 34.99 (sD 20.17) nmol/l, 
Table 1. Distribution of maternal characteristics by folate status: the Hokkaido Study on Environment and Children's Health 2002-2012, Japan† (Numbers and percentages; $n$ 15266)

Annual income (million JPY)

Occupation

Tobacco smoking (cigarette sticks/d)

ETS at home
ETS at workplace

Combined ETS exposure at home and workplace

Plasma cotinine status $(\mathrm{nmol} / \mathrm{l})$

Alcohol intake (frequency)

$\begin{array}{ll} & \text { Monthly } \\ & \text { Weekly } \\ & \text { Daily } \\ \text { Folic acid supplements use } & \text { No } \\ & \text { Yes } \\ \text { Other nutritional supplements use } & \text { No } \\ & \text { Yes } \\ \text { Region } & \text { Central } \\ & \text { South } \\ & \text { East } \\ \text { Year of enrolment } & \text { Other regions } \\ & 2002-2004 \\ & 2005-2007 \\ \text { Season of the year at enrolment } & 2008-2010 \\ & 2011-2012 \\ & \text { Spring } \\ & \text { Summer } \\ & \text { Autumn } \\ & \text { Winter }\end{array}$

JPY, Japanese Yen; ETS, environmental tobacco smoke.

${ }^{*} P<0.050 ;{ }^{* *} P<0.010 ;{ }^{* \star *} P<0.001$.

$\dagger P$ values were derived from Pearson's $X^{2}$ tests and Fisher's exact tests. All percentages are row percentages. Values may not add up to $100 \%$ due to missing values.

$P=0 \cdot 028$, respectively (Fig. 2). Users of FA supplements were likely to be those with chronic inter-current medical conditions, those who had fertility treatments and those who were also users of other nutritional supplements; $7 \cdot 0 \%$ of FA users started intake more than 3 months before conception. Another $8.0 \%$ started 1 month before conception, whereas the majority 


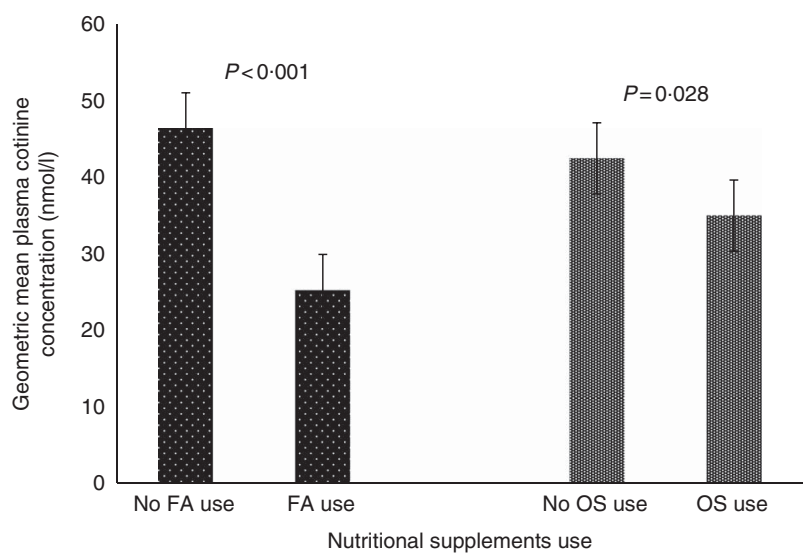

Fig. 2. Mean plasma cotinine concentrations by nutritional supplements use among participants: the Hokkaido Study on Environment and Children's Health, 2002-2012, Japan. FA, folic acid; OS, other nutritional supplements.

(more than 60.0\%) started use following confirmation of pregnancy. The average frequency of use per week was three times. Multivitamins reported were found to contain various doses of FA in the range of $100-200 \mu \mathrm{g} /$ tablet (data not shown).

In the regression model, the value of McFadden's pseudo $R^{2}$ pooled over the $m=10$ imputed data sets was $8.7 \%$. In Table 2, the demographic determinants of low folate status are shown: as lower maternal age (adjusted OR (AOR) 1.48; $95 \% \mathrm{CI}$ $1 \cdot 32,1 \cdot 66, P<0 \cdot 001$ ), lower educational level (AOR 1.27; $95 \%$ CI $1.17,1.39, P<0.001$ ), lower annual income (AOR 1.11; $95 \%$ CI $1.01,1.22, P=0.024)$, residing in the south and eastern regions (AOR 1.25; $95 \%$ CI 1.14, 1.38, $P<0.001$ ) and (AOR 1.15; $95 \%$ CI 1.05, 1.25, $P=0.003)$, respectively. Being enrolled into the study between 2005 and 2007 was associated with an increase in the risk of low folate status (AOR 1.23; 95\% CI 1.12, $1 \cdot 35, P<0 \cdot 001$ ), whereas recruitment between 2008 and 2010 reduced the likelihood of having low folate status (AOR 0.81; $95 \%$ CI $0.73,0.90, P<0.001)$, respectively. Being enrolled during summer, autumn and winter were associated with higher likelihood of low folate status (AOR 1.12; $95 \%$ CI 1.02, 1.24, $P=0.023$ ), (AOR 1.13 ; $95 \%$ CI $1.02,1.25, P=0.015)$ and (AOR $1 \cdot 13$; $95 \%$ CI $1.01,1 \cdot 27, P=0.037$ ), respectively. Lower BMI (AOR 0.84; 95\% CI 0.74, 0.94, $P=0.006$ ) and unemployment were associated with risk reduction (AOR 0.87; 95\% CI 0.80, $0 \cdot 94, P=0 \cdot 001)$.

Lifestyle factors that reduced the odds of low folate status were the use of FA supplements (AOR 0.19; $95 \%$ CI 0.17, 0.22, $P<0.001$ ), other nutritional supplements (AOR 0.55; 95\% CI $0.48,0.64, P<0.001)$ and weekly alcohol consumption (AOR $0.75 ; 95 \%$ CI $0.62,0.90, P=0.003)$. Lifestyle factors that increased the odds of low folate status were active cigarette smoking and ETS exposure. Smoking $<10$ cigarette sticks/d was associated with increased odds (AOR 1.42; $95 \%$ CI 1.23, 1.64, $P<0.001$ ), whereas smoking between 10 and 19 cigarette sticks/d was associated with an increased risk (AOR 2.28; $95 \%$ CI 1.92, 2.71, $P<0.001)$. However, smoking $\geq 20$ cigarette sticks/d was not statistically significant, $P_{\text {trend }}<0 \cdot 001$. Exposure to ETS at home increased the odds of low folate status (AOR $1 \cdot 23 ; 95 \%$ CI $1.13,1.34, P<0.001)$, and exposure to ETS at the workplace also increased the odds of low folate status (AOR 1.16; $95 \%$ CI 1.02, 1.31, $P=0 \cdot 026$ ).

Using plasma cotinine concentrations to classify active and passive exposure to tobacco products, Table 3 shows that participants with plasma cotinine levels between 1.19 and $65.21 \mathrm{nmol} / 1$ were 1.20 times more likely to have low folate status (AOR 1.20; $95 \%$ CI 1.10, 1.31, P<0.001), whereas those with levels $>65.21 \mathrm{nmol} / 1$ had a 2 -fold increase in risk (AOR $1.91 ; 95 \%$ CI $1 \cdot 70,2 \cdot 14, P<0 \cdot 001) ; P_{\text {trend }}<0 \cdot 001$.

\section{Discussion}

To our knowledge, this is the first report to present robust information on demographic and lifestyle predictors of folate status in a relatively large cohort of pregnant Japanese women. The majority $(73.8 \%)$ of participants had optimal first-trimester folate status. Only $0.5 \%$ had serum folate concentrations $<6.80 \mathrm{nmol} / \mathrm{l}$, a level clinically considered a negative folate balance, whereas $25.7 \%$ of the population had marginal folate status. Lower tolerance limit of $8.47 \mathrm{nmol} / 1$ implies a negative folate balance for this population. Our findings contrast those from Tokyo where $>50.0 \%$ of the study population of pregnant women had low folate status.

\section{Demographic predictors of folate status}

Low folate status was associated with younger maternal age, higher BMI, lower educational level and lower annual income. Cigarette smoking rate is on the increase among young Japanese women, and a quest to achieve a lower BMI via dieting is in vogue among women of reproductive age. These factors may invariably compromise nutritional status including folate among younger women ${ }^{(4,17)}$. Micronutrient deficiencies including folate in overweight/obese people have been reported by some previous studies ${ }^{(18)}$. Possible mechanisms postulated have been decreased dietary intake, current cigarette smoking and possible low serum/plasma concentrations as a result of increased intravascular volume ${ }^{(19)}$. Consistent with our findings, socio-economic status has been reported to influence folate intake among Japanese workers ${ }^{(20)}$. In addition, educational attainment was reported to do so in Belgium ${ }^{(21)}$ and Australia $^{(22)}$. In the USA, older maternal age, higher education and higher income status have been reported to predict the use of FA supplements ${ }^{(23)}$. In this study, these factors might have favoured higher folate status. Other demographic factors associated with suboptimal folate status have been reported from other countries, and these include household size ${ }^{(24)}$, season of the year ${ }^{(25)}$, rural residence ${ }^{(26)}$ and region ${ }^{(27)}$. We observed that residing in the southern and eastern regions and seasons of the year were associated with the risk of low folate status.

Traditionally, most Japanese women are full-time house wives. This may explain why the unemployed had lower risk. Working women are likely to skip their meals and may prefer fast foods as reported among children of working women ${ }^{(28)}$. Of note here is that employment status was broadly classified. Further exploration based on job types may shed more insight on this observation. 
Table 2. Estimated effects of demographic characteristics and lifestyle factors on folate status: the Hokkaido Study on Environment and Children's Health 2002-2012, Japant

(Numbers and percentages; adjusted odds ratios (AOR) and $95 \%$ confidence intervals; $n$ 15266)

\begin{tabular}{|c|c|c|c|c|c|c|c|c|}
\hline & \multirow[b]{3}{*}{ Categories } & \multicolumn{4}{|c|}{ Folate status (nmol/l) } & \multirow[b]{3}{*}{ AOR } & \multirow[b]{3}{*}{$95 \% \mathrm{Cl}$} & \multirow[b]{3}{*}{$P_{\text {trend }}$} \\
\hline & & \multicolumn{2}{|c|}{ Suboptimal $(<13.60)$} & \multicolumn{2}{|c|}{ Optimal $(\geq 13 \cdot 60)$} & & & \\
\hline & & $n 3995$ & $\%(26 \cdot 2)$ & $n 11271$ & $\%(73.8)$ & & & \\
\hline \multirow[t]{2}{*}{ Age (years) } & $<25$ & 685 & $38 \cdot 16$ & 1110 & $61 \cdot 84$ & 1.48 & $1.32,1.66^{\star \star \star}$ & \\
\hline & $\geq 25$ & 3137 & 24.54 & 9647 & 75.46 & 1.00 & Ref. & \\
\hline \multirow[t]{2}{*}{ BMI $\left(\mathrm{kg} / \mathrm{m}^{2}\right)$} & $<25$ & 3362 & $25 \cdot 65$ & 9746 & $74 \cdot 35$ & 0.84 & $0.74,0.95^{\star \star}$ & \\
\hline & $\geq 25$ & 460 & 29.91 & 1078 & 70.09 & 1.00 & Ref. & \\
\hline \multirow[t]{2}{*}{ Parity } & Nulliparous & 1576 & $26 \cdot 34$ & 4407 & 73.66 & 0.96 & $0.88,1.05$ & \\
\hline & Parous & 2163 & $26 \cdot 92$ & 5872 & 73.08 & 1.00 & Ref. & \\
\hline \multirow[t]{2}{*}{ Educational level } & High school & 2277 & 31.06 & 5054 & 68.94 & 1.27 & $1 \cdot 17,1 \cdot 39^{\star \star \star}$ & \\
\hline & At least college & 1613 & 21.40 & 5925 & 78.60 & 1.00 & Ref. & \\
\hline \multirow[t]{2}{*}{ Income (million JPY) } & $<5$ & 2421 & 28.08 & 6202 & 71.92 & $1 \cdot 11$ & $1.01,1.22^{\star}$ & \\
\hline & $\geq 5$ & 897 & $21 \cdot 86$ & 3207 & $78 \cdot 14$ & 1.00 & Ref. & \\
\hline \multirow{2}{*}{ Occupation } & Ūnemployed & 1599 & 24.74 & 4865 & $75 \cdot 26$ & 0.87 & $0.80,0.94^{\star *}$ & \\
\hline & Employed & 2396 & $27 \cdot 22$ & 6406 & $72 \cdot 78$ & 1.00 & Ref. & \\
\hline \multirow[t]{4}{*}{ Region } & Central & 1549 & 23.06 & 5169 & $76 \cdot 94$ & 1.00 & Ref. & NS \\
\hline & South & 1094 & 30.48 & 2495 & 69.52 & 1.25 & $1 \cdot 14,1 \cdot 38^{\star \star \star}$ & \\
\hline & East & 1304 & $27 \cdot 37$ & 3461 & $72 \cdot 63$ & $1 \cdot 15$ & $1.05,1.25^{\star \star}$ & \\
\hline & Other regions & 48 & 24.74 & 146 & $75 \cdot 26$ & 0.75 & $0.53,1.07$ & \\
\hline \multirow[t]{4}{*}{ Year of enrolment } & 2002-2004 & 1305 & $28 \cdot 23$ & 3318 & 71.77 & 1.00 & Ref. & $<0.010$ \\
\hline & 2005-2007 & 1710 & $30 \cdot 26$ & 3941 & $69 \cdot 74$ & $1 \cdot 23$ & $1 \cdot 12,1 \cdot 35^{\star \star \star}$ & \\
\hline & 2008-2010 & 804 & 19.79 & 3259 & $80 \cdot 21$ & 0.81 & $0.73,0.90^{\star \star \star}$ & \\
\hline & 2011-2012 & 176 & 18.95 & 753 & 81.05 & 0.83 & $0.69,1.00$ & \\
\hline \multirow[t]{4}{*}{ Season of the year at enrolment } & Spring & 1041 & $27 \cdot 04$ & 2809 & $72 \cdot 96$ & 1.00 & Ref. & $<0.010$ \\
\hline & Summer & 1004 & 26.99 & 2716 & 73.01 & $1 \cdot 12$ & $1.02,1.24^{*}$ & \\
\hline & Autumn & 642 & $26 \cdot 49$ & 1782 & 73.51 & $1 \cdot 13$ & $1.02,1 \cdot 25^{\star}$ & \\
\hline & Winter & 1308 & $24 \cdot 81$ & 3964 & $75 \cdot 19$ & $1 \cdot 13$ & $1.01,1.27^{\star}$ & \\
\hline \multirow[t]{2}{*}{ Folic acid supplements use } & No & 3746 & $31 \cdot 29$ & 8224 & $68 \cdot 71$ & 1.00 & Ref. & \\
\hline & Yes & 249 & 7.55 & 3047 & 92.45 & 0.19 & $0.17,0.22^{\star \star \star}$ & \\
\hline \multirow[t]{2}{*}{ Other nutritional supplements use $\neq$} & No & 3734 & $26 \cdot 76$ & 10222 & 73.24 & 1.00 & Ref. & \\
\hline & Yes & 261 & $19 \cdot 92$ & 1049 & $80 \cdot 08$ & 0.55 & $0.48,0.64^{\star \star \star}$ & \\
\hline \multirow[t]{4}{*}{ Alcohol intake } & No & 2002 & $24 \cdot 76$ & 6425 & 73.72 & 1.00 & Ref. & NS \\
\hline & Monthly & 1620 & $28 \cdot 98$ & 3970 & $71 \cdot 02$ & $1 \cdot 21$ & $1 \cdot 11,1 \cdot 31^{\star \star \star}$ & \\
\hline & Weekly & 163 & 22.54 & 560 & 77.46 & 0.75 & $0.62,0.90^{\star \star}$ & \\
\hline & Daily & 210 & $24 \cdot 17$ & 659 & $75 \cdot 83$ & 0.91 & $0.76,1.08$ & \\
\hline \multirow[t]{4}{*}{ Tobacco smoking (number of cigarette sticks/d) } & No & 3308 & 24.40 & 10251 & $75 \cdot 60$ & 1.00 & Ref. & $<0.001$ \\
\hline & $<10$ & 351 & 36.00 & 624 & 64.00 & 1.42 & $1.23,1.64^{\star \star \star}$ & \\
\hline & $10-19$ & 301 & $47 \cdot 78$ & 329 & $52 \cdot 22$ & $2 \cdot 28$ & $1.92,2 \cdot 71^{\star \star \star}$ & \\
\hline & $\geq 20$ & 35 & 34.31 & 67 & 65.69 & $1 \cdot 18$ & $0.77,1.81$ & \\
\hline \multirow[t]{2}{*}{ ETS at home } & No & 1203 & $20 \cdot 87$ & 4560 & $79 \cdot 13$ & 1.00 & Ref. & \\
\hline & Yes & 2792 & 29.38 & 6711 & 70.62 & 1.23 & $1.13,1.34^{\star \star \star}$ & \\
\hline \multirow[t]{2}{*}{ ETS at workplace } & No & 394 & $25 \cdot 75$ & 1136 & $74 \cdot 25$ & 1.00 & Ref. & \\
\hline & Yes & 3601 & $26 \cdot 22$ & 10135 & 73.78 & $1 \cdot 16$ & $1 \cdot 02,1 \cdot 31^{*}$ & \\
\hline
\end{tabular}

Ref., referent values; JPY, Japanese Yen; ETS, environmental tobacco smoke.

${ }^{*} P<0.050 ;{ }^{* *} P<0.010 ;{ }^{* * *} P<0.001$.

† Regression model adjusted for maternal age, parity, BMI, educational level, annual income, occupation, geographical region, year of enrolment into the study, season of the year at enrolment, nutritional supplements use, alcohol intake and active and passive smoking. McFadden's pseudo $R^{2}=8.7 \%$. All percentages are row percentages. Values may not add up to $100 \%$ due to missing values.

‡ Other nutritional supplements used included multivitamins, trace elements, herbs, proteins, ginseng and energy drinks.

\section{Unfavourable lifestyle predictors of folate status}

We report self-reported active cigarette smoking and ETS exposure as major modifiable unfavourable predictors of folate status. Although we could not demonstrate a dose-response pattern in the odds, especially among heavy smokers during pregnancy, this may probably be related to a small subgroup size. Using plasma cotinine as a biomarker, the risk of low folate status increased in a dose-response pattern. Contrary to this result, another study in Tokyo found no lifestyle habits as risk factors for suboptimal folate status ${ }^{(8)}$. However, our result is consistent with reports from other developed countries, where lifestyle factors are commonly observed as predictors of folate status. Folate-depleting effects of active smoking and ETS exposure have been reported ${ }^{(29,30-35)}$. Possible biological mechanisms of folate depletion in active and passive smokers include decreased intake ${ }^{(29,33)}$, inactivating effects of organic nitrites, cyanates and nitrous oxide on circulating folates ${ }^{(34,36)}$ and direct effects of oxidative stress or increased folate turnover ${ }^{(31,37)}$. We observed lower mean plasma cotinine concentrations among nutritional supplements users. Nutritional supplements users are more likely to practise healthy lifestyles. 
Table 3. Estimated effects of active and passive cigarette smoking based on plasma cotinine concentrations on folate status: the Hokkaido Study on Environment and Children's Health 2002-2012, Japant

(Numbers and percentages; adjusted odds ratios (AOR) and $95 \%$ confidence intervals; $n$ 15266)

\begin{tabular}{|c|c|c|c|c|c|c|c|c|}
\hline \multirow[b]{3}{*}{ Smoking status } & \multirow[b]{3}{*}{ Plasma cotinine levels $(\mathrm{nmol} / \mathrm{l})$} & \multicolumn{4}{|c|}{ Folate status (nmol/l) } & \multirow[b]{3}{*}{ AOR } & \multirow[b]{3}{*}{$95 \% \mathrm{Cl}$} & \multirow[b]{3}{*}{$P_{\text {trend }}$} \\
\hline & & \multicolumn{2}{|c|}{ Suboptimal $(<13.60)$} & \multicolumn{2}{|c|}{ Optimal $(\geq 13 \cdot 60)$} & & & \\
\hline & & $n 3995$ & $\%(26.17)$ & $n 11271$ & $\%(73.83)$ & & & \\
\hline Non-smoker & $<1.19$ & 1164 & $19 \cdot 82$ & 4710 & $80 \cdot 18$ & 1.00 & Ref. & $<0.001$ \\
\hline ETS exposed & $1 \cdot 19-65 \cdot 21$ & 1940 & $27 \cdot 27$ & 5173 & $72 \cdot 73$ & 1.20 & $1 \cdot 10,1 \cdot 31^{\star \star *}$ & \\
\hline Active smoker & $>65.21$ & 891 & $39 \cdot 10$ & 1388 & $60 \cdot 90$ & 1.91 & $1 \cdot 70,2 \cdot 14^{\star \star \star}$ & \\
\hline
\end{tabular}

Ref., referent values; ETS, environmental tobacco smoke.

Levels of significance: ${ }^{* *} P<0.001$.

† Regression model adjusted for maternal age, parity, BMI, educational level, annual income, occupation, geographical region, year of enrolment into the study, season of the year at enrolment, nutritional supplements use, alcohol intake and active and passive smoking. McFadden's pseudo $R^{2}=8.5 \%$. All percentages are row percentages. Values may not add up to $100 \%$ due to missing values.

\section{Favourable lifestyle predictors of folate status}

FA supplements use is the major modifiable predictor of optimal folate status. This report further confirms the welldocumented role of FA supplements in improving folate status. Other nutritional supplements used also correlated positively with folate status, probably because most multivitamins also contain FA. Other nutritional supplements used included multivitamins, trace elements, herbs, proteins, ginseng and energy drinks. Over-the-counter multivitamins used contained various doses of FA in the range of $100-200 \mu \mathrm{g} / \mathrm{tablet}$ according to the brand names reported by study participants - the majority of whom were recruited between 2002 and 2010. However, lately, the FA content seems to have been increased by drug makers (up to $480 \mu \mathrm{g} /$ tablet). This may reflect in our findings of an increase in mean folate concentrations of participants enrolled from 2010 and beyond and a reduction in the risk of having low folate status. In this study, the majority of FA supplement users did not use it because of pregnancy. Those who used it for prenatal purpose started only after confirming that they were pregnant. This information may impact on the crucial periconceptional period for prevention of NTD. Within Japan, some smaller studies outside Hokkaido did report that using FA supplements increased blood folate concentrations more than using dietary sources of folate only. They also observed that Japanese women of reproductive age do not meet the daily RDA of $440 \mu \mathrm{g}$ for folate ${ }^{(5,7,8,38,39)}$. Although the Japanese Government has recommended that women of reproductive age or those who plan to become pregnant should take $400 \mu \mathrm{g} / \mathrm{d}$ of FA supplements, scholars have reported that the levels of awareness and compliance with the recommendations are still low ${ }^{(5,40)}$. Furthermore, across the Asian subregion, prenatal FA supplements use is not a routine prenatal care practice ${ }^{(41)}$. Our findings are similar to other reports emerging from China, Malaysia and Indonesia. Of these three, mandatory fortification is legislated only in Indonesia ${ }^{(42-44)}$. Internationally, studies from other developed countries without food fortification policies are reporting increasing incidence of suboptimal folate concentrations ${ }^{(21,45,46)}$. Our result on the role of alcoholic beverage consumption on folate status is consistent with a previous study in the Czech Republic, where moderate beer consumption correlated with higher plasma folate ${ }^{(47)}$. Conversely, chronic heavy alcohol consumption is associated with folate deficiency via numerous mechanisms ${ }^{(48)}$. We stand with the universal recommendation that pregnant women should abstain from consuming alcoholic beverages, because of adverse fetal effects ${ }^{(49)}$.

\section{Strengths and limitations}

This study is the first to utilise a large population of pregnant Japanese women who were recruited early enough within the stage of embryonic neurulation and organogenesis. Epidemiologically, the study identified demographic and lifestyle determinants of folate status at this critical stage of neural tube formation. Identifying modifiable lifestyle factors as favourable and unfavourable determinants can lay a sound foundation for public health intervention policies. All information about the type or brand name of nutritional supplements used as well as the timing and duration of use were self-reported, and hence the risk of bias. However, nutritional supplements use and smoking status were validated by biomarkers to avoid misclassification bias. For instance, the difference observed in folate biomarker concentrations among FA users and non-users was an indication of valid self-reported use. In addition, comparable results were obtained with plasma cotinine and self-reported cigarette smoking or ETS exposure. Serum folate was used as an indicator of folate status. Erythrocyte folate signifies tissue folate reserves and is not subject to dietary fluctuations exhibited by serum/plasma folate concentrations, thus making it a more reliable choice. However, because erythrocyte folate determination is more complex, the serum folate assay was preferred to conduct this large epidemiological study; two previous studies have justified its use in epidemiological studies ${ }^{(50,51)}$. This study involved only women who presented at the designated healthcare facilities and consented to participate; therefore, it may not be representative of the general population. Finally, our findings are more of statistical correlations and not in any way signifying causality. Future randomised-controlled trials using erythrocyte folate and known dosages of FA supplements may be more informative. 


\section{Implications}

The implication of active and passive tobacco smoking for the determination of folate status is of public health importance because an increasing prevalence of tobacco smoking among younger Japanese women is being reported ${ }^{(52)}$. Optimal first-trimester folate status is central in this subpopulation. It may be helpful to consider policies that could improve folate status in this group. Mandatory food fortification with FA might be a great precautionary measure. Although there are emerging controversies about prenatal FA exposure and epigenetic effects $^{(53)}$, the folate-depleting effects of tobacco smoke may constitute a huge public health challenge in the prevention of NTD and other birth defects in Japan. Although this Hokkaido cohort data recorded only eight (0.04\%) cases of isolated NTD, the national rate is the second highest in developed countries after Germany.

In conclusion, demographic and lifestyle factors likely predict the folate status of Hokkaido women. Active cigarette smoking and ETS exposure are the major modifiable unfavourable predictors of folate status, whereas the use of FA supplements and FA-containing multivitamins are the major favourable predictors. FA supplementation may correct the folate deficits associated with tobacco smoking.

\section{Acknowledgements}

The authors express their profound gratitude to the study participants and to all of personnel of various hospitals and clinics that collaborate with the study: Keiai Hospital, Endo Kikyo Maternity Clinic, Shiroishi Hospital, Memuro Municipal Hospital, Aoba Ladies Clinic, Obihiro-Kyokai Hospital, Akiyama Memorial Hospital, Sapporo Medical University Hospital, Hokkaido University Hospital, Kitami Red Cross Hospital, Hoyukai Sapporo Hospital, Gorinbashi Hospital, Hashimoto Clinic, Asahikawa Medical College Hospital, Hakodate Central General Hospital, Ohji General Hospital, Nakashibetsu Municipal Hospital, Sapporo Tokushukai Hospital, Asahikawa Red Cross Hospital, Wakkanai City Hospital, Kushiro Rosai Hospital, Sapporo-Kosei General Hospital, Shibetsu City General Hospital, Nikko Memorial Hospital, Sapporo City General Hospital, Kohnan Hospital, Hakodate City Hospital, Hokkaido Monbetsu Hospital, Tenshi Hospital, Hakodate Goryoukaku Hospital, Nakamura Hospital, Kin-ikyo Sapporo Hospital, Kitami Lady's Clinic, Engaru- Kosei General Hospital, Kushiro Red Cross Hospital, Nayoro City General Hospital and Obihiro-Kosei General Hospital.

Supported by Grant-in-Aid for Scientific Research from the Japanese Ministry of Health, Labour and Welfare (grant no. 717L000103) and Japan Society for the Promotion of Science (JSPS KAKENHI grant no. 25253050).

T. A. Y.: directly involved in the data interpretation and writing of the final manuscript; A. A.: funding preparation; S. S.: blood cotinine analysis; T. A. Y., C. M. and E. Y.: data analysis; K. I., H. M., T. E. and K. S.: data collection; T. I.: funding preparation; S. K.: sample collection; H. G., T. B.: data cleaning; T. B.: cotinine measurement; and R. K.: principal investigator.

There are no conflicts of interest.

\section{References}

1. Grant A, Chalmers I, Lucock MD, et al. (1991) Vitamins to prevent neural tube defects. Lancet 338, 894-895.

2. Cragan JD, Roberts HE, Edmonds LD, et al. (1995) Surveillance for anencephaly and spina bifida and the impact of prenatal diagnosis - United States, 1985-1994. MMWR CDC Surveill Summ 44, 1-13.

3. Flour Fortification Initiative (2011) Fifteen years of fortifying with folic acid reduces birth defects; averts healthcare expenses. http://www.ffinetwork.org/about/stay_informed/ publications/documents/FolicAcidBackground.pdf (accessed February 2016).

4. Takimoto $\mathrm{H} \&$ Tamura $\mathrm{T}$ (2006) Increasing trend of spina bifida and decreasing birth weight in relation to declining body-mass index of young women in Japan. Med Hypotheses 67, 1023-1026.

5. Kondo A, Kamihira O \& Ozawa H (2009) Neural tube defects: prevalence, etiology and prevention. Int J Urol 16, 49-57.

6. Kondo A, Kamihira O, Shimosuka Y, et al. (2005) Awareness of the role of folic acid, dietary folate intake and plasma folate concentration in Japan. J Obstet Gynaecol Res 31, 172-177.

7. Mito N, Takimoto H, Umegaki K, et al. (2007) Folate intakes and folate biomarker profiles of pregnant Japanese women in the first trimester. Eur J Clin Nutr 61, 83-90.

8. Matsuzaki M, Haruna M, Ota E, et al. (2008) Dietary folate intake, use of folate supplements, lifestyle factors, and serum folate levels among pregnant women in Tokyo, Japan. J Obstet Gynaecol Res 34, 971-979.

9. Ihara $\mathrm{H}$, Watanabe $\mathrm{T}$, Aoki $\mathrm{Y}$, et al. (2009) Dietary folate intake and serum folate status in Japanese women of childbearing age. J Anal Bio-Sci 32, 5 .

10. Yila TA, Sasaki S, Miyashita C, et al. (2012) Effects of maternal 5,10-methylenetetrahydrofolate reductase C677T and A1298C Polymorphisms and tobacco smoking on infant birth weight in a Japanese population. J Epidemiol 22, 91-102.

11. Kishi R, Sasaki S, Yoshioka E, et al. (2011) Cohort profile: the Hokkaido study on environment and children's health in Japan. Int J Epidemiol 40, 611-618.

12. Kishi R, Kobayashi S, Ikeno T, et al. (2013) Ten years of progress in the Hokkaido birth cohort study on environment and children's health: cohort profile - updated 2013. Environ Health Prev Med 18, 429-450.

13. Bayer Diagnostics (1998) Diagnostics B Package Insert: ADVIA Centaur Assay Manual. Tarrytown, NY: Bayer Diagnostics, Revision AT.

14. Sujaku K, Ogiwara T \& Kawasaki Y (2006) Determination of vitamin $\mathrm{B}_{12}$ and folate by ADVIA Centaur. J Anal Bio-Sci 29, 235-242.

15. Sauberlich HE (1999) Folate (FA, pteroylmonoglutamic acid, folacin). In Laboratory Tests for the Assessment of Nutritional Status, 2nd ed., pp. 103-134 [I Wolinsky, editor]. Washington, DC: CRC Press.

16. Sasaki S, Braimoh TS, Yila TA, et al. (2011) Self-reported tobacco smoke exposure and plasma cotinine levels during pregnancy - a validation study in Northern Japan. Sci Total Environ 412-413, 114-118.

17. Takimoto H, Yoshiike N, Kaneda F, et al. (2004) Thinness among young Japanese women. Am J Public Health 94 , 1592-1595.

18. Damms-Machado A, Weser G \& Bischoff SC (2012) Micronutrient deficiency in obese subjects undergoing low calorie diet. Nutr J 11, 34 .

19. Aasheim ET, Hofso D, Hjelmesaeth J, et al. (2008) Vitamin status in morbidly obese patients: a cross-sectional study. $\mathrm{AmJ}$ Clin Nutr 87, 362-369. 
20. Miyaki K, Song Y, Taneichi S, et al. (2013) Socioeconomic status is significantly associated with the dietary intakes of folate and depression scales in Japanese workers (J-HOPE Study). Nutrients 5, 565-578.

21. Vandevijvere S, Amsalkhir S, Van Oyen H, et al. (2012) Determinants of folate status in pregnant women: results from a national cross-sectional survey in Belgium. Eur J Clin Nutr 66, 1172-1177.

22. Gall S, Seal J, Taylor R, et al. (2012) Folate status and sociodemographic predictors of folate status, among a national cohort of women aged 26-36 in Australia, 2004-2006. Aust NZ J Public Health 36, 421-426.

23. Branum AM, Bailey R \& Singer BJ (2013) Dietary supplement use and folate status during pregnancy in the United States. J Nutr 143, 486-492.

24. Thoradeniya $\mathrm{T}$, Wickremasinghe $\mathrm{R}$, Ramanayake $\mathrm{R}$, et al. (2006) Low folic acid status and its association with anaemia in urban adolescent girls and women of childbearing age in Sri Lanka. Br J Nutr 95, 511-516.

25. Hao L, Ma J, Stampfer MJ, et al. (2003) Geographical, seasonal and gender differences in folate status among Chinese adults. J Nutr 133, 3630-3635.

26. Garcia-Casal MN, Osorio C, Landaeta M, et al. (2005) High prevalence of folic acid and vitamin $\mathrm{B}_{12}$ deficiencies in infants, children, adolescents and pregnant women in Venezuela. Eur J Clin Nutr 59, 1064-1070.

27. Zhao Y, Hao L, Zhang L, et al. (2009) Plasma folate status and dietary folate intake among Chinese women of childbearing age. Matern Child Nutr 5, 104-116.

28. Gaina A, Sekine M, Chandola T, et al. (2009) Mother employment status and nutritional patterns in Japanese junior high schoolchildren. Int J Obes (Lond) 33, 753-757.

29. Mathews F, Yudkin P, Smith RF, et al. (2000) Nutrient intakes during pregnancy: the influence of smoking status and age. $J$ Epidemiol Community Health 54, 17-23.

30. Trobs M, Renner T, Scherer G, et al. (2002) Nutrition, antioxidants, and risk factor profile of nonsmokers, passive smokers and smokers of the Prevention Education Program (PEP) in Nuremberg, Germany. Prev Med 34, 600-607.

31. Ulvik A, Ebbing M, Hustad S, et al. (2010) Long- and shortterm effects of tobacco smoking on circulating concentrations of B vitamins. Clin Chem 56, 755-763.

32. Mannino DM, Mulinare J, Ford ES, et al. (2003) Tobacco smoke exposure and decreased serum and red blood cell folate levels: data from the Third National Health and Nutrition Examination Survey. Nicotine Tob Res 5, 357-362.

33. Ortega RM, Requejo AM, Lopez-Sobaler AM, et al. (2004) Smoking and passive smoking as conditioners of folate status in young women. $J$ Am Coll Nutr 23, 365-371.

34. Erdemir EO \& Bergstrom J (2007) Effect of smoking on folic acid and vitamin $\mathrm{B}_{12}$ after nonsurgical periodontal intervention. J Clin Periodontol 34, 1074-1081.

35. Vardavas CI, Linardakis MK, Hatzis CM, et al. (2008) Smoking status in relation to serum folate and dietary vitamin intake. Tob Induc Dis $\mathbf{4}, 8$.

36. Ozerol E, Ozerol I, Gokdeniz R, et al. (2004) Effect of smoking on serum concentrations of total homocysteine, folate, vitamin $\mathrm{B}_{12}$, and nitric oxide in pregnancy: a preliminary study. Fetal Diagn Ther 19, 145-148.
37. Yanbaeva DG, Dentener MA, Creutzberg EC, et al. (2007) Systemic effects of smoking. Chest 131, 1557-1566.

38. Kondo A, Shimosuga Y, Oguchi H, et al. (2008) Folic acid reduces the risk of neural tube defects: awareness and folate intake among pregnant women in 2006. Hinyokika Kiyo $\mathbf{5 4}$, 537-542.

39. Kondo A, Asada Y, Shibata K, et al. (2011) Dietary folate intakes and effects of folic acid supplementation on folate concentrations among Japanese pregnant women. $J$ Obstet Gynaecol Res 37, 331-336.

40. Japan Ministry of Health (2000) Department of Maternal and Child Health, Bureau of Children and Families [BoCaF], Tokyo: Information on Promoting Intake of FA in Order to Reduce Children Afflicted with Neural Tube Defects Among Young Women Who Are Capable of Becoming Pregnant. http://www1.mhlw.go.jp/houdou/1212/h1228-1_18. html (accessed April 2016).

41. Oi S (2003) Current status of prenatal management of fetal spina bifida in the world: worldwide cooperative survey on the medico-ethical issue. Childs Nerv Syst 19, 596-599.

42. Ren A, Zhang L, Li Z, et al. (2006) Awareness and use of folic acid, and blood folate concentrations among pregnant women in northern China - an area with a high prevalence of neural tube defects. Reprod Toxicol 22, 431-436.

43. Khor GL, Duraisamy G, Loh SP, et al. (2006) Dietary and blood folate status of Malaysian women of childbearing age. Asia Pac J Clin Nutr 15, 341-349.

44. Green TJ, Skeaff CM, Venn BJ, et al. (2007) Red cell folate and predicted neural tube defect rate in three Asian cities. Asia Pac J Clin Nutr 16, 269-273.

45. Brough L, Rees GA, Crawford MA, et al. (2009) Social and ethnic differences in folic acid use preconception and during early pregnancy in the UK: effect on maternal folate status. J Hum Nutr Diet 22, 100-107.

46. McNulty B, Pentieva K, Marshall B, et al. (2011) Women's compliance with current folic acid recommendations and achievement of optimal vitamin status for preventing neural tube defects. Hum Reprod 26, 1530-1536.

47. Mayer O Jr, Simon J \& Rosolova H (2001) A population study of the influence of beer consumption on folate and homocysteine concentrations. Eur J Clin Nutr 55, 605-609.

48. Halsted CH, Villanueva JA, Devlin AM, et al. (2002) Metabolic interactions of alcohol and folate. J Nutr 132, 2367S-2372S.

49. International Center for Alcohol Policies (ICAP) (1995) ICAP Blue Book 10. Drinking and pregnancy. http://www.icap.org/PolicyTools/ICAPBlueBook/BlueBook Modules/10DrinkingandPregnancy/tabid/171/Default.aspx (accessed February 2016).

50. Galloway M \& Rushworth L (2003) Red cell or serum folate? Results from the National Pathology Alliance benchmarking review. J Clin Pathol 56, 924-926.

51. Drogan D, Klipstein-Grobusch K, Wans S, et al. (2004) Plasma folate as marker of folate status in epidemiological studies: the European Investigation into Cancer and Nutrition (EPIC)Potsdam study. Br J Nutr 92, 489-496.

52. Takimoto H, Yokoyama T, Yoshiike N, et al. (2005) Increase in low-birth-weight infants in Japan and associated risk factors, 1980-2000. J Obstet Gynaecol Res 31, 314-322.

53. Dolinoy DC (2007) Epigenetic gene regulation: early environmental exposures. Pharmacogenomics 8, 5-10. 